\title{
Deep Neuromuscular Blockade Combined with Low Pneumoperitoneum Pressure for Nociceptive Recovery After Major Laparoscopic Gastrointestinal Surgery: Study Protocol for a Randomized Controlled Trial
}

\author{
Yu-qin Long $\mathbb{D}^{1,2}$ \\ Xi-sheng Shan ${ }^{1,2}$ \\ Xiao-mei Feng ${ }^{3}$ \\ Hong Liu $\mathbb{1}^{4}$ \\ Fu-hai Ji (D) ${ }^{1,2}$ \\ Ke Peng (D) ${ }^{1,2}$
}

'Departments of Anesthesiology, First Affiliated Hospital of Soochow University, Suzhou, Jiangsu, People's Republic of China; ${ }^{2}$ Institute of Anesthesiology, Soochow University, Suzhou, Jiangsu, People's Republic of China; ${ }^{3}$ Department of Anesthesiology, University of Utah Health, Salt Lake City, UT, USA;

${ }^{4}$ Department of Anesthesiology and Pain Medicine, University of California Davis Health, Sacramento, CA, USA
Correspondence: Ke Peng

Department of Anesthesiology, First

Affiliated Hospital of Soochow University,

I88 Shizi Street, Suzhou, Jiangsu, 215006,

People's Republic of China

Tel +86-159-62I5-5989

Email pengke0422@163.com
Purpose: Patients undergoing major laparoscopic surgery often experience significant pain and postoperative nausea and vomiting (PONV). Deep neuromuscular block (NMB) improves surgical conditions and facilitates the application of low intra-abdominal pressure (IAP), which may be beneficial for these patients. This study is designed to determine the effects of deep NMB combined with low IAP, as compared to moderate NMB combined with standard IAP, on patients' nociceptive recovery after major laparoscopic gastrointestinal surgery.

Study Design and Methods: This single-center randomized controlled trial will include 220 patients scheduled for major laparoscopic gastrointestinal surgery (lasts for $\geq 90$ minutes). Patients will be randomly assigned, with a 1:1 ratio, into a deep NMB + low IAP group (train of four $=0$, post-tetanic count $=1-3, \mathrm{IAP}=8 \mathrm{mmHg}$ ) and a moderate $\mathrm{NMB}+$ standard IAP group (train of four $=1-3$, IAP $=12 \mathrm{mmHg}$ ). If the surgical workspace is inadequate, the surgeons can request a step increase of $1 \mathrm{mmHg}$ in IAP during 3-min intervals. The upper limit of IAP will be set at $15 \mathrm{mmHg}$. Postoperative recovery will be assessed using the postoperative quality recovery scale (PQRS). The primary outcome of this trial is the PQRS nociceptive recovery (including pain and PONV) at postoperative day (POD) 1 . The secondary outcomes include recovery in other PQRS domains at POD 1, and recovery in all PQRS domains in a post-anesthesia care unit, at POD 3 in the surgical wards, at hospital discharge, and at postoperative 30 days. For the sample size estimation, 110 patients in each group (220 in total) would be needed to detect an absolute increase rate of $20 \%$ in the PQRS nociceptive domain in the deep NMB + low IAP group at POD 1.

Discussion: This study investigates the effects of deep NMB combined with low IAP on postoperative PQRS nociceptive recovery in patients undergoing major laparoscopic gastrointestinal surgery. We expect that this deep NMB + low IAP strategy would improve postoperative pain and PONV following major laparoscopic gastrointestinal surgery.

Keywords: major laparoscopic surgery, pneumoperitoneum pressure, neuromuscular block, postoperative quality recovery scale, randomized controlled trial

\section{Introduction}

Over the decades, laparoscopic surgical procedures have been widely performed worldwide. Many studies have demonstrated the safety and benefits of laparoscopic surgery as compared to open surgery. ${ }^{1-5}$ During a laparoscopy, carbon dioxide 
$\left(\mathrm{CO}_{2}\right)$ is insufflated into the abdominal cavity to create a pneumoperitoneum. A high intraoperative intraabdominal pressure (IAP) during pneumoperitoneum is associated with increased postoperative morbidity. ${ }^{6-10}$ While clinical guidelines on the pneumoperitoneum for laparoscopic surgery recommend the use of the lowest IAP for an adequate surgical workspace, ${ }^{11}$ it is still a common practice in many clinical settings that a standard IAP of $12-15 \mathrm{mmHg}$ is used throughout surgery. ${ }^{12}$ A recent meta-analysis showed that deep neuromuscular blockade (NMB) improved the surgical conditions and facilitated the application of low IAP during laparoscopic surgery, when compared to moderate NMB. ${ }^{13}$

Although laparoscopy is minimally invasive compared to laparotomy, patients undergoing major laparoscopic surgical procedures still experience significant pain and postoperative nausea and vomiting (PONV). ${ }^{14-16}$ In a recent study of patients undergoing laparoscopic colorectal surgery, an individualized pneumoperitoneum pressure strategy (ie, deep NMB, abdominal wall stretching, and targeting the lowest IAP) improved patients' physiological recovery on the Postoperative Quality Recovery Scale (PQRS), reduced intraoperative cough and movement, and attenuated inflammation during postoperative 3 days. ${ }^{17}$ While these findings are promising, this study is underpowered to test postoperative pain and PONV. In addition, patients' recovery data were assessed in the early postoperative days only.

Therefore, the present study aims to determine the effects of a deep NMB combined with low IAP strategy on patients' recovery at emergence from general anesthesia, during the hospitalization, and at 30 days after major laparoscopic gastrointestinal surgery. The primary focus of this study is postoperative recovery in the PQRS nociceptive domain including postoperative pain and PONV, assessed at postoperative day (POD) 1 . We expect that the results of this trial would provide evidence for clinical use of the deep NMB combined with low IAP strategy, for the purpose of improving postoperative recovery in patients undergoing major laparoscopic gastrointestinal surgery.

\section{Methods}

\section{Study Design}

This single-blind, randomized, parallel-controlled, singlecenter trial was approved by the Ethic Committee of First Affiliated Hospital of Soochow University (Approval
No. 2020-520). The protocol was registered at the Chinese Clinical Trial Registry (http://www.chictr.org.cn, identifier: ChiCTR2000034957). The First Affiliated Hospital of Soochow University will be responsible for patient enrollment, allocation, intervention, follow-up, and data analysis. This trial will be conducted in accordance with the Declaration of Helsinki. A Steering Committee will monitor the source data. This study protocol follows the Standard Protocol Items: Recommendations for Interventional Trials (SPIRIT) statement. $^{18}$

\section{Participants}

A total of 220 eligible patients who are ready to undergo major laparoscopic gastrointestinal surgery (lasts for $\geq 90$ minutes) will be randomly assigned to receive either a deep neuromuscular block combined with a low intraabdominal pressure (the deep NMB + low IAP group) or a moderate neuromuscular block combined with a standard pressure (the moderate NMB + standard IAP group). A preoperative investigator who is not involved in the following study will screen the patients' admission records to identify potentially eligible participants. Written informed consent will be obtained from each subject. The study flow diagram is shown in Figure 1.

\section{Inclusion Criteria}

The inclusion criteria are: 1) age $>18$ years; 2) American Society of Anesthesiologists (ASA) physical status IIII; 3) scheduled for major laparoscopic gastrointestinal surgery under general anesthesia; and 4) provision of written informed consent.

\section{Exclusion Criteria}

The exclusion criteria are: 1) emergency or unplanned surgery; 2) pregnancy; 3) severe cardiopulmonary, cerebrovascular, hepatic, or renal disease; 4) immune system or neuromuscular disease; 5) inability to cooperate or complete the study questionnaires; or 6) known allergies to the medications used in this study.

\section{Randomization and Blinding}

A research assistant who is not involved in the subsequent study will generate the random sequence by using an online randomization tool, with a 1:1 ratio and block sizes of 4 and 6 (https://www.sealedenvelope.com/simplerandomiser/v1/lists). The randomization will be stratified according to sex. The details of allocation and intervention plans will be sealed in the identical opaque envelopes with 


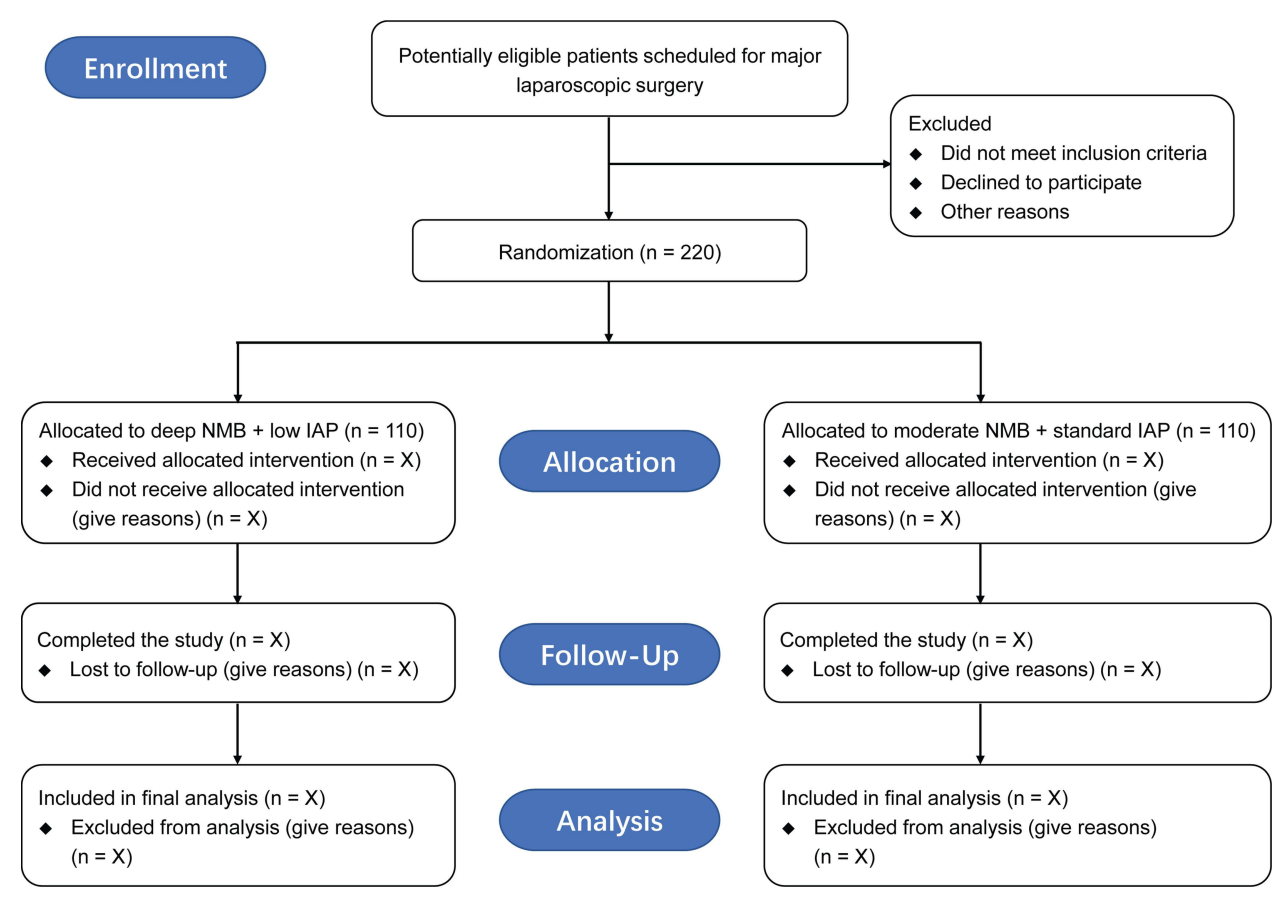

Figure I Study flow diagram.

the labels of serial numbers of patients. When a patient meets the eligibility criteria, the preoperative screener will send the corresponding envelope to an attending anesthesiologist. The attending anesthesiologist will then open the envelope and provide intraoperative anesthesia management according to the intervention plans.

Because of the differences between the two groups in terms of NMB degree and pneumoperitoneum pressure levels, it is not possible to mask the attending anesthesiologists to the group assignment. However, the anesthesiologists will not take part in patient recruitment, data collection, outcome assessment, or statistical analysis. To mask the surgeons to the group assignment, the pneumoperitoneum insufflator screen will be covered by a dark curtain. Patients, surgeons, preoperative and postoperative evaluators, and other healthcare providers will be blinded to the group allocation until the completion of final analysis.

\section{Study Interventions}

The schedule for enrollment, intervention, and assessment according to SPIRIT 2013 statement is shown in Table 1. The details of study interventions in the deep NMB + low IAP group and the moderate NMB + standard IAP group are shown in Table 2.
For the deep NMB + low IAP group, an initial dose of rocuronium $1.2 \mathrm{mg} / \mathrm{kg}$ will be administered for tracheal intubation, followed by additional doses of $0.15 \mathrm{mg} / \mathrm{kg}$ to achieve a train of four (TOF) of 0 and a post-tetanic count (PTC) of 1-3 during surgery. Neuromuscular transmission will be monitored at the adductor pollicis muscle. When no response is detected with $2-\mathrm{Hz}$ TOF stimulations, the PTC will be measured with a 5 -second $50-\mathrm{Hz}$ tetanic stimulus every 5 minutes. ${ }^{19,20}$ After $\mathrm{CO}_{2}$ inflation through the trocars, an IAP of $12 \mathrm{mmHg}$ will be maintained for 5 min, and then the IAP will be decreased stepwise to 8 $\mathrm{mmHg}$. If the surgical workspace is not adequate, the surgeons can request a step increase of $1 \mathrm{mmHg}$ in IAP during 3-min intervals. The upper limit of IAP will be set at $15 \mathrm{mmHg}$. After the surgery, sugammadex $4 \mathrm{mg} / \mathrm{kg}$ will be used for NMB reversal before tracheal extubation.

For the moderate NMB + standard IAP group, an initial dose of rocuronium $0.6 \mathrm{mg} / \mathrm{kg}$ will be used, followed by additional doses of $0.15 \mathrm{mg} / \mathrm{kg}$ to achieve an intraoperative TOF of $1-3$. The TOF was measured every 5 minutes. The IAP will be set at $12 \mathrm{mmHg}$ throughout surgery. The surgeons can also request a higher IAP as that in the deep NMB + low IAP group, with the upper limit of IAP at $15 \mathrm{mmHg}$. Neostigmine $30 \mu \mathrm{g} / \mathrm{kg}$ with atropine 20 $\mu \mathrm{g} / \mathrm{kg}$ will be used for NMB reversal when the TOF $\geq 3$ is reached. 
Table I Schedule of Enrollment, Interventions, and Assessments

\begin{tabular}{|c|c|c|c|c|c|c|c|c|c|}
\hline \multirow[b]{3}{*}{ Timepoint } & \multicolumn{9}{|c|}{ Study Period } \\
\hline & \multirow{2}{*}{$\begin{array}{c}\text { Enrollment } \\
\text { POD -I }\end{array}$} & \multirow{2}{*}{$\begin{array}{c}\text { Allocation } \\
\text { 2h before } \\
\text { surgery }\end{array}$} & \multicolumn{6}{|c|}{ Post-Allocation } & \multirow{2}{*}{$\begin{array}{c}\text { Close- } \\
\text { Out } \\
\text { POD } \\
30\end{array}$} \\
\hline & & & $\begin{array}{l}\text { During } \\
\text { surgery }\end{array}$ & $\begin{array}{l}\text { PACU I5 } \\
\text { min }\end{array}$ & $\begin{array}{c}\text { PACU } 40 \\
\text { min }\end{array}$ & $\begin{array}{c}\text { POD } \\
\text { I }\end{array}$ & $\begin{array}{c}\text { POD } \\
3\end{array}$ & $\begin{array}{l}\text { Hospital } \\
\text { discharge }\end{array}$ & \\
\hline $\begin{array}{l}\text { Enrolment } \\
\text { Eligibility screening } \\
\text { Informed consent } \\
\text { Randomization } \\
\text { Allocation }\end{array}$ & $\begin{array}{l}x \\
x \\
x\end{array}$ & $\mathrm{X}$ & & & & & & & \\
\hline $\begin{array}{l}\text { Interventions } \\
\text { Deep NMB + low IAP } \\
\text { Moderate NMB + } \\
\text { standard IAP }\end{array}$ & & & $\begin{array}{l}x \\
x\end{array}$ & & & & & & \\
\hline $\begin{array}{l}\text { Assessments } \\
\text { PQRS } \\
\text { Adverse events } \\
\text { Complications } \\
\text { Mortality }\end{array}$ & & $x$ & $x$ & $x$ & $x$ & $\begin{array}{l}x \\
x\end{array}$ & $\begin{array}{l}x \\
x\end{array}$ & $\begin{array}{l}x \\
x \\
x\end{array}$ & $\begin{array}{l}x \\
x\end{array}$ \\
\hline
\end{tabular}

Notes: According to SPIRIT 2013 statement: defining standard protocol items for clinical trials.

Abbreviations: NMB, neuromuscular block; IAP, intra-abdominal pressure; PQRS, Postoperative Quality of Recovery Scale; PACU, post-anesthesia care unit; POD, postoperative day.

\section{Anesthesia Management}

Patients will fast for 6 hours, without premedication. According to the random code, patients will be randomly assigned to receive the allocated interventions. In the

Table 2 Details of Study Interventions

\begin{tabular}{|l|l|l|}
\hline & Deep NMB + Low IAP & $\begin{array}{l}\text { Moderate NMB + } \\
\text { Standard IAP }\end{array}$ \\
\hline $\begin{array}{l}\text { Muscle } \\
\text { relaxant }\end{array}$ & $\begin{array}{l}\text { Rocuronium } 1.2 \mathrm{mg} / \mathrm{kg}+ \\
\text { repeat doses of } 0.15 \mathrm{mg} / \\
\mathrm{kg}\end{array}$ & $\begin{array}{l}\text { Rocuronium } 0.6 \mathrm{mg} / \mathrm{kg}+ \\
\text { repeat doses of } 0.15 \mathrm{mg} / \\
\mathrm{kg}\end{array}$ \\
\hline TOF & 0 & $\mathrm{I}$ to 3 \\
\hline PTC & 1 to 3 & $\mathrm{NA}$ \\
\hline $\begin{array}{l}\text { Intra- } \\
\text { abdominal } \\
\text { pressure }\end{array}$ & $\begin{array}{l}12 \mathrm{mmHg} \text { for } 5 \mathrm{~min}, \text { and } \\
\text { stepwise to } 8 \mathrm{mmHg}\end{array}$ & $\begin{array}{l}12 \mathrm{mmHg} \text { throughout } \\
\text { surgery }\end{array}$ \\
\hline Reversal & Sugammadex $4 \mathrm{mg} / \mathrm{kg}$ & $\begin{array}{l}\text { Neostigmine } 30 \mu \mathrm{g} / \mathrm{kg}+ \\
\text { atropine } 20 \mu \mathrm{g} / \mathrm{kg}, \text { when } \\
\text { TOF } \geq 3\end{array}$ \\
\hline
\end{tabular}

Abbreviations: NMB, neuromuscular block; IAP, intra-abdominal pressure; TOF, train of four; PTC, post-tetanic count. operating room, the monitoring includes radial arterial blood pressure, electrocardiography, pulse oximetry, bispectral index (BIS, Aspect Medical Systems, Newton, MA), surgical pleth index (SPI, S/5 $5^{\mathrm{TM}}$ Anaesthesia Monitor, GE Healthcare, Helsinki, Finland), and neuromuscular blockade (TOF-Watch-SXTM, Organon Teknika, Oss, Netherlands).

General anesthesia will be induced with sufentanil 0.4 $\mu \mathrm{g} / \mathrm{kg}$ and propofol $2 \mathrm{mg} / \mathrm{kg}$. Tracheal intubation will be facilitated with rocuronium. Patients' lungs will be mechanically ventilated with a tidal volume of $8-10 \mathrm{~mL} / \mathrm{kg}$, frequency of $12-18$ breaths $/ \mathrm{min}$, and $50 \%$ oxygen in air, adjusted to maintain end-tidal $\mathrm{CO}_{2}$ values within 35-45 mmHg. After anesthesia induction, patients will receive intravenous dexamethasone $5 \mathrm{mg}$ and dexmedetomidine infusion of $0.4 \mu \mathrm{g} / \mathrm{kg} / \mathrm{h}$ until skin closure. Anesthesia will be maintained with $1-3 \%$ sevoflurane inhalation, titrated to BIS values within 45-55. To provide adequate intraoperative analgesia, additional doses of sufentanil will be used based on the changes in heart rate (HR), mean arterial pressure (MAP), and SPI. Previous studies have shown that adequate intraoperative analgesia was achieved at SPI values within $20-50 .^{21,22}$ When a patient shows signs of insufficient 
analgesia, ie, SPI values $>50$ for $>20$ seconds, tachycardia (HR $>100$ beats/min) or hypertension (MAP increase $>$ $20 \%$ of the baseline value), an additional bolus of sufentanil $0.1 \mu \mathrm{g} / \mathrm{kg}$ will be administered. ${ }^{23}$ If there is sufficient depth of anesthesia and analgesia (BIS values $<55$ and SPI values $<50$ ), hypertension will be treated with intravenous urapidil $5 \mathrm{mg}$, and tachycardia will be treated with intravenous esmolol $20 \mathrm{mg}$. Hypotension (a decrease in MAP > 20\% of the baseline value) will be treated with intravenous phenylephrine $50 \mu \mathrm{g}$, and bradycardia (HR $<50$ beats/min) will be treated with intravenous atropine $0.5 \mathrm{mg}$. Fluid management will be left to the discretion of the attending anesthesiologists. Nasopharyngeal temperature will be maintained within $36-37^{\circ} \mathrm{C}$ by using a warming blanket and an infusion heating device.

At the end of the surgery, patients will receive intravenous ondansetron $8 \mathrm{mg}$ for preventing PONV. After extubation, patients will be transferred to a post-anesthesia care unit (PACU). Patients will receive standard monitoring and nasal oxygen supplementation in the PACU. Postoperative pain will be treated with patientcontrolled intravenous analgesia (PCIA) in the PACU and in the surgical wards. The PCIA device contains sufentanil $1 \mu \mathrm{g} / \mathrm{mL}$ in $100 \mathrm{~mL}$ normal saline, with a background infusion of $1 \mathrm{~mL} / \mathrm{h}$, a bolus dose of $2 \mathrm{~mL}$, and a lockout time of 5 minutes. Patients will be encouraged to press the demand button for postoperative pain relief.

The Modified Aldrete Score will be assessed every 5 minutes, with a score $\geq 9$ indicating readiness for discharge from the PACU to the surgical wards. ${ }^{24}$ The hospital discharge criteria for our patients consists of tolerance of oral intake, return of bowel function, sufficient pain control, adequate mobility, and absence of complications. ${ }^{25}$ All surgical procedures, anesthesia and analgesia management, and perioperative care will be provided by the same medical team. The flow diagram of perioperative management is depicted in Figure 2.
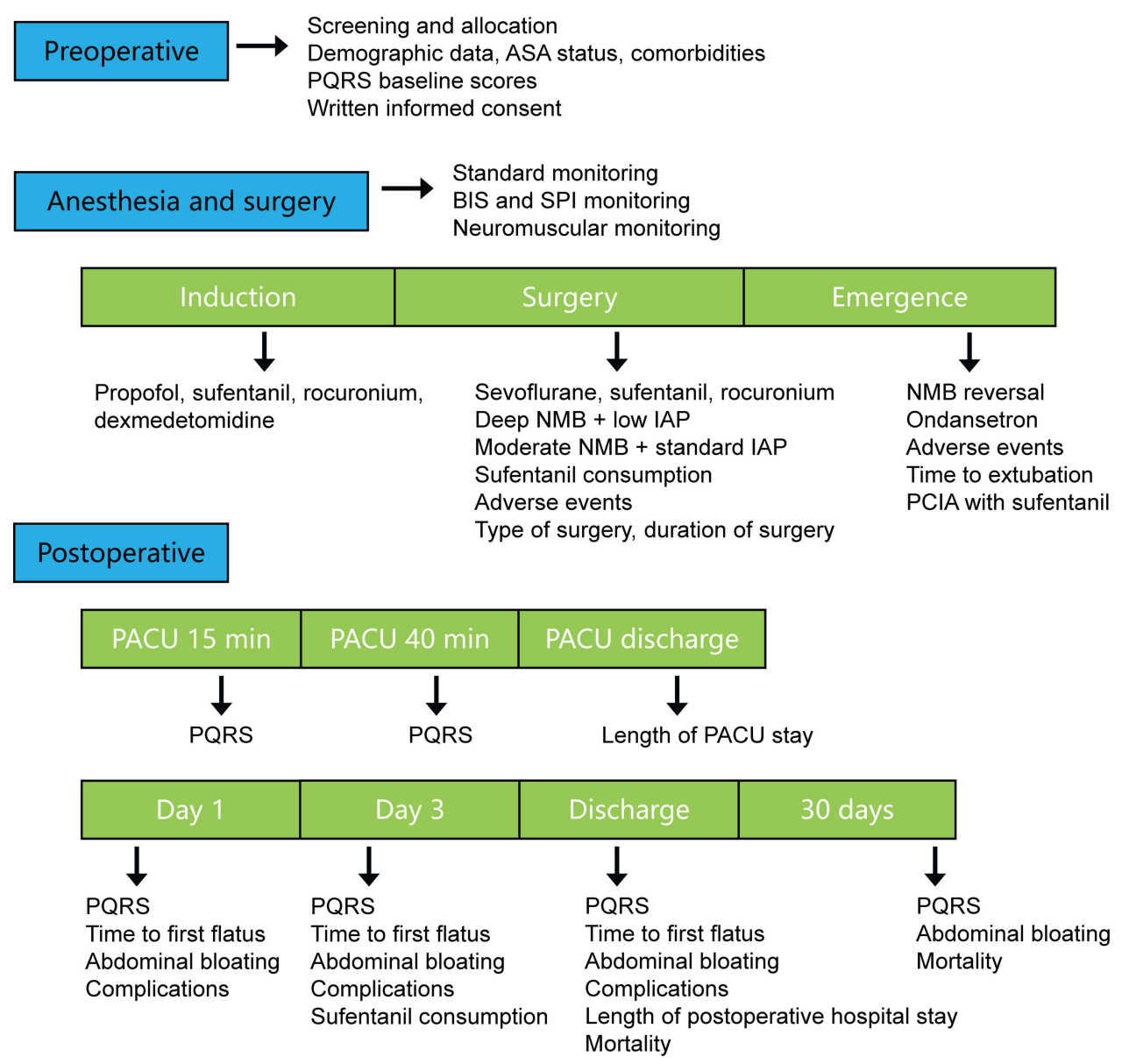

Figure 2 Study plan and anesthesia management.

Abbreviations: PQRS, Postoperative Quality of Recovery Scale; BIS, bispectral index; SPI, surgical pleth index; NMB, neuromuscular block; IAP, intra-abdominal pressure; $\mathrm{PCIA}$, patient-controlled intravenous analgesia; PACU, post-anesthesia care unit. 


\section{Postoperative Quality Recovery Scale}

The PQRS is a validated and easy to use verbal survey tool (www.postopqrs.com), which was developed to assess postoperative recovery at $15 \mathrm{~min}$ (early in the PACU), 40 min (at the point of PACU discharge), postoperative day 1, postoperative day 3 , and 3 months postoperatively. ${ }^{26}$ Later, many studies have validated the use of PQRS to assess postoperative recovery. ${ }^{17,27-29}$ The PQRS consists of six domains: physiologic, nociceptive, emotive, activities of daily living, cognitive, and overall patient perspective. ${ }^{26}$ For each patient, the baseline PQRS values will be obtained before surgery. To evaluate the patient's recovery, multiple items in each domain will be assessed and compared with the baseline scores. At each assessment timepoint, recovery in each domain is defined as a return to the baseline values or better, and the overall recovery is defined as recovery in all domains.

\section{Primary and Secondary Outcomes}

The primary outcome of this study is the recovery in the PQRS nociceptive domain (pain and PONV) at POD 1. The secondary outcomes include recovery in other PQRS domains at POD 1, and recovery in all PQRS domains at 15 and $40 \mathrm{~min}$ in the PACU, at POD 3, at hospital discharge, and at postoperative 30 days.

\section{Data Collection}

The preoperative screener will collect patients' demographic data, baseline characteristics, and PONV risk factors (including female gender, non-smoking, history of motion sickness or PONV, and postoperative opioid use). ${ }^{30}$ An anesthesia nurse will collect intraoperative IAP values, type of surgery, sufentanil consumption, duration of surgery, time to extubation, and adverse events.

An independent postoperative observer will collect patients' postoperative data, including PCAI sufentanil consumption, abdominal bloating, time to first flatus, postoperative complications (atrial fibrillation, cardiac arrest, heart block, myocardial infarction, atelectasis, acute respiratory distress syndrome, pneumonia, respiratory failure, delirium, transient ischemic attack, stroke, acute kidney injury, renal failure, renal replacement therapy, deep vein thrombosis, wound infection, sepsis, reoperation for hemorrhage, anastomotic leak, and paralytic ileus), length of PACU stay, length of postoperative hospital stay, and in-hospital mortality. Abdominal bloating will be categorized as mild (no interruption on oral intake or sleep), moderate (delayed oral intake or use of laxatives), or severe (need for gastrointestinal decompression). ${ }^{31}$ The definitions of postoperative complications are shown in Supplementary File 1.

An independent research investigator who is experienced in the use of PQRS survey tool will collect the PQRS scores before surgery, at $15 \mathrm{~min}$ and $40 \mathrm{~min}$ in the PACU, at POD 1 and POD 3 in the surgical wards, and at hospital discharge. In addition, the PQRS scores, abdominal bloating, and survival status at postoperative 30 days will be collected via telephone.

\section{Adverse Events and Patient Safety}

Any adverse events related to the study interventions or not (such as allergic reaction, intra-abdominal organ injury, major intraoperative bleeding, and cardiovascular events) will be recorded, and the attending anesthesiologists and attending surgeons will immediately provide relevant treatment to ensure the patient's safety. In case of a serious adverse event, the principal investigator of the study and the Steering Committee will be immediately notified to unmask the group allocation and decide whether the patient should be withdrawn from this study.

\section{Sample Size Calculation}

The sample size calculation is based on the primary outcome (ie, recovery of nociception) using the PASS software (version 11.0.7, NCSS, LCC, Kaysville, UT). In the previous study, approximately $30 \%$ of patients undergoing laparoscopic colorectal surgery showed recovery in the PQRS nociceptive domain at POD $1 .{ }^{17}$ We expect that $50 \%$ of patients receiving deep $\mathrm{NMB}$ and low IAP would have their nociceptive recovery, with an absolute increase rate of $20 \%$. To detect such a difference with an $\alpha$ level of 0.05 and a power of $80 \%$, we estimate that 94 participants are needed per group. Considering a possible dropout rate of $15 \%, 110$ patients will be enrolled in each group.

\section{Statistical Analysis}

All analyses will be performed based on the intention-totreat principle. Patients were be included in the statistical analysis if they were randomized to receive the corresponding interventions (deep or moderate NMB and low or standard pneumoperitoneum pressure) no matter whether the IAP changes during surgery. We do not have plans for imputation of missing data or interim analysis. Data normality will be checked using the Shapiro-Wilk 
test. Continuous variables will be presented as mean \pm standard deviation or median (interquartile ranges), and analyzed using the unpaired $t$ test, repeated measures analysis of variance, or Mann-Whitney $U$-test, as appropriate. Categorical variables will be presented as number (percentages) of patients and analyzed using the chisquared test or Fisher's exact test. The incidence of recovery over time in each domain will be analyzed by using a generalised linear mixed model. For the primary outcome, the effect size (mean difference or relative risk with $95 \%$ confidence intervals) will be reported. All data analyses will be performed using the GraphPad Prism software (version 7.00, GraphPad Software Inc., San Diego, CA). A two-sided $P<0.05$ will be considered statistically significant.

\section{Discussion}

In this randomized controlled trial of patients undergoing major laparoscopic gastrointestinal surgery, we intend to evaluate the effects of deep NMB in combination with low IAP on postoperative recovery, when compared with moderate NMB combined with standard IAP. Our primary hypothesis is that this deep NMB + low IAP strategy would result in a better PQRS nociceptive recovery, that is, an improvement in postoperative pain and PONV. The second hypothesis is that patients' recovery in other domains and the overall recovery would also be improved both in the early postoperative period and up to 30 days after surgery. The implementation and reporting of this trial will follow the Consolidated Standards of Reporting Trials (CONSORT) guidelines. ${ }^{32}$

In the common practice of laparoscopic surgery, patients receive a moderate $\mathrm{NMB}$, a standard IAP, and neostigmine for NMB reversal. $^{33}$ Compared with a moderate NMB strategy, a deep NMB strategy facilitated the use of low IAP and improved the quality of surgical field. ${ }^{34}$ However, several studies reported that the use of deep NMB alone without the application of a low IAP did not improve surgical conditions, postoperative pain, or quality of recovery after laparoscopic surgery. ${ }^{35-37}$ A previous study suggested that the combination of deep NMB and low IAP reduced shoulder pain after laparoscopic hysterectomy. ${ }^{38}$ A recent study showed that a low IAP did not lead to lower postoperative pain scores but reduced fentanyl requirement and the incidence of PONV in the recovery room after laparoscopic cholecystectomy. ${ }^{39}$ To date, the effects of deep NMB + low IAP strategy on postoperative nociceptive recovery including pain and
PONV after major laparoscopic surgery are still inconclusive.

The use of neostigmine for reversal of moderate NMB is according to the daily clinical practice. For a deep NMB, however, it is not possible to reverse the block with neostigmine, and sugammadex should be used instead. Rocuronium-induced NMB can be reversed by sugammadex rapidly, regardless of the depth of block. While sugammadex has obvious advantages over neostigmine in terms of fast onset, predictable reversal, and low risk of residual block, both agents are safe to use, with a very low incidence of serious adverse events. ${ }^{33}$ Compared to neostigmine, sugammadex has been shown to improve postoperative recovery at $40 \mathrm{~min}$ after surgery, but not at POD 1 or POD $3 .^{29}$ Thus, the use of sugammadex or neostigmine is not a major contributor to postoperative recovery beyond the anesthesia emergence period. In this study, we will use the combination of deep NMB and low IAP to reduce the congestion pressure and promote postoperative recovery.

This study has several strengths. First, to the best of our knowledge, this will be the first randomized controlled trial with an adequate power to investigate the effects of deep NMB combined with low IAP on postoperative PQRS nociceptive recovery in patients undergoing major laparoscopic gastrointestinal surgery. Second, each patient's postoperative recovery will be comprehensively assessed using the PQRS, a tool that has been validated in the literature. ${ }^{26-29,40}$ Next, this study will collect postoperative recovery data after emergence from general anesthesia and during hospital stay, as well as recovery outcomes up to 30 days after surgery.

This study also has some limitations. First, the attending anesthesiologists are aware of the group allocation, as they provide different levels of NMB. However, the PQRS data will be collected by an independent research investigator not involved in the perioperative medical care, and patients will be blinded to the allocation throughout the study. In this regard, the risk of bias will be low. Second, this is a single-center trial, so the results of this study may not be fully generalizable to other centers. Next, we include relatively healthy patients with ASA status I-III, and thus the effects of deep NMB combined with low IAP in patients who are older and/or with more significant comorbidities need further investigations.

In conclusion, this trial aims to determine the effects of deep NMB combined with low IAP, as compared to moderate NMB combined with standard IAP, on postoperative 
pain and PONV in patients undergoing major laparoscopic gastrointestinal surgery. We will report the results in accordance with the CONSORT checklist. ${ }^{32}$ We expect that this deep NMB combined with low IAP strategy would improve postoperative recovery in the PQRS nociceptive domain, as well as in other PQRS domains.

\section{Author Contributions}

All authors made a significant contribution to the work reported, whether that is in the conception, study design, execution, acquisition of data, analysis and interpretation, or in all these areas; took part in drafting, revising or critically reviewing the article; gave final approval of the version to be published; have agreed on the journal to which the article has been submitted; and agree to be accountable for all aspects of the work.

\section{Funding}

This work will be supported by the Science and Technology Development Plan Clinical Trial Project (SLT201909 to FHJ) and Jiangsu Provincial Medical Youth Talents Program (QNRC2016741 to KP).

\section{Disclosure}

The authors report no conflicts of interest in this work.

\section{References}

1. Krielen P, Stommel MWJ, Pargmae P, et al. Adhesion-related readmissions after open and laparoscopic surgery: a retrospective cohort study (SCAR update). Lancet. 2020;395(10217):33-41. doi:10.1016/ S0140-6736(19)32636-4

2. Clinical Outcomes of Surgical Therapy Study Group. A comparison of laparoscopically assisted and open colectomy for colon cancer. $N \mathrm{Engl}$ J Med. 2004;350(20):2050-2059.

3. Kang SB, Park JW, Jeong SY, et al. Open versus laparoscopic surgery for mid or low rectal cancer after neoadjuvant chemoradiotherapy (COREAN trial): short-term outcomes of an open-label randomised controlled trial. Lancet Oncol. 2010;11(7):637-645. doi:10.1016/ S1470-2045(10)70131-5

4. Liu F, Huang C, Xu Z, et al. Morbidity and mortality of laparoscopic vs open total gastrectomy for clinical stage I gastric cancer: the CLASS02 multicenter randomized clinical trial. JAMA Oncol. 2020;6(10):1590-1597. doi:10.1001/jamaoncol.2020.3152

5. van Vugt JL, Reisinger KW, Derikx JP, Boerma D, Stoot JH. Improving the outcomes in oncological colorectal surgery. World $J$ Gastroenterol. 2014;20(35):12445-12457. doi:10.3748/wjg.v20. i35.12445

6. Sammour T, Mittal A, Loveday BP, et al. Systematic review of oxidative stress associated with pneumoperitoneum. Br J Surg. 2009;96 (8):836-850. doi:10.1002/bjs.6651

7. Brokelman WJ, Lensvelt M, Borel Rinkes IH, Klinkenbijl JH, Reijnen MM. Peritoneal changes due to laparoscopic surgery. Surg Endosc. 2011;25(1):1-9. doi:10.1007/s00464-010-1139-2
8. Schwarte LA, Scheeren TW, Lorenz C, De Bruyne F, Fournell A. Moderate increase in intraabdominal pressure attenuates gastric mucosal oxygen saturation in patients undergoing laparoscopy. Anesthesiology. 2004;100(5):1081-1087. doi:10.1097/00000542200405000-00009

9. Cheng ZJ, Wang YB, Chen L, Gong JP, Zhang W. Effects of different levels of intra-abdominal pressure on the postoperative hepatic function of patients undergoing laparoscopic cholecystectomy: a systematic review and meta-analysis. Surg Laparosc Endosc Percutan Tech. 2018;28(5):275-281. doi:10.1097/SLE.0000000 000000525

10. Rohloff M, Cicic A, Christensen C, Maatman TK, Lindberg J, Maatman TJ. Reduction in postoperative ileus rates utilizing lower pressure pneumoperitoneum in robotic-assisted radical prostatectomy. J Robot Surg. 2019;13(5):671-674. doi:10.1007/s11701-018-00915-w

11. Neudecker J, Sauerland S, Neugebauer E, et al. The European Association for Endoscopic Surgery clinical practice guideline on the pneumoperitoneum for laparoscopic surgery. Surg Endosc. 2002;16(7):1121-1143. doi:10.1007/s00464-001-9166-7

12. Hypolito $\mathrm{OH}$, Azevedo JL, de Lima Alvarenga Caldeira FM, et al. Creation of pneumoperitoneum: noninvasive monitoring of clinical effects of elevated intraperitoneal pressure for the insertion of the first trocar. Surg Endosc. 2010;24(7):1663-1669. doi:10.1007/s00464009-0827-2

13. Bruintjes MH, van Helden EV, Braat AE, et al. Deep neuromuscular block to optimize surgical space conditions during laparoscopic surgery: a systematic review and meta-analysis. Br J Anaesth. 2017;118 (6):834-842. doi:10.1093/bja/aex116

14. Dewinter G, Coppens S, Van de Velde M, et al. Quadratus lumborum block versus perioperative intravenous lidocaine for postoperative pain control in patients undergoing laparoscopic colorectal surgery: a prospective, randomized, double-blind controlled clinical trial. Ann Surg. 2018;268(5):769-775. doi:10.1097/SLA.0000000000002888

15. Bakri MH, Ismail EA, Ibrahim A. Comparison of dexmedetomidine and dexamethasone for prevention of postoperative nausea and vomiting after laparoscopic cholecystectomy. Korean J Anesthesiol. 2015;68(3):254-260. doi:10.4097/kjae.2015.68.3.254

16. White PF, Tang J, Hamza MA, et al. The use of oral granisetron versus intravenous ondansetron for antiemetic prophylaxis in patients undergoing laparoscopic surgery: the effect on emetic symptoms and quality of recovery. Anesth Analg. 2006;102(5):1387-1393. doi:10.1213/01.ane.0000208967.94601.cd

17. Díaz-Cambronero O, Mazzinari G, Flor Lorente B, et al. Effect of an individualized versus standard pneumoperitoneum pressure strategy on postoperative recovery: a randomized clinical trial in laparoscopic colorectal surgery. Br J Surg. 2020;107(12):1605-1614. doi:10.1002/ bjs. 11736

18. Chan AW, Tetzlaff JM, Gotzsche PC, et al. SPIRIT 2013 explanation and elaboration: guidance for protocols of clinical trials. $B M J$. 2013;346:e7586. doi:10.1136/bmj.e7586

19. Dhonneur G, Kirov K, Motamed C, et al. Post-tetanic count at adductor pollicis is a better indicator of early diaphragmatic recovery than train-of-four count at corrugator supercilii. $\mathrm{Br} J$ Anaesth. 2007;99(3):376-379. doi:10.1093/bja/aem124

20. Murphy GS. Neuromuscular monitoring in the perioperative period. Anesth Analg. 2018;126(2):464-468. doi:10.1213/ANE.0000000 000002387

21. Gruenewald M, Willms S, Broch O, Kott M, Steinfath M, Bein B. Sufentanil administration guided by surgical pleth index vs standard practice during sevoflurane anaesthesia: a randomized controlled pilot study. Br J Anaesth. 2014;112(5):898-905. doi:10.1093/bja/ aet 485

22. Ryu KH, Kim JA, Ko DC, Lee SH, Choi WJ. Desflurane reduces intraoperative remifentanil requirements more than sevoflurane: comparison using surgical pleth index-guided analgesia. $\mathrm{Br} J$ Anaesth. 2018;121(5):1115-1122. doi:10.1016/j.bja.2018.05.064 
23. Hou YH, Shi WC, Cai S, et al. Effect of intravenous lidocaine on serum interleukin-17 after video-assisted thoracic surgery for non-small-cell lung cancer: a randomized, double-blind, placebo-controlled trial. Drug Des Devel Ther. 2021;15:3379-3390. doi:10.2147/DDDT.S316804

24. Hetta DF, Elgalaly NA, Hetta HF, Fattah Mohammad MA. Preoperative Duloxetine to improve acute pain and quality of recovery in patients undergoing modified radical mastectomy: a dose-ranging randomized controlled trial. $J$ Clin Anesth. 2020;67:110007. doi:10.1016/j.jclinane.2020.110007

25. Fiore JF Jr, Browning L, Bialocerkowski A, Gruen RL, Faragher IG, Denehy L. Hospital discharge criteria following colorectal surgery: a systematic review. Colorectal Dis. 2012;14(3):270-281. doi:10.1111/j.1463-1318.2010.02477.x

26. Royse C, Newman S, Chung F, et al. Development and feasibility of a scale to assess postoperative recovery: the post-operative quality recovery scale. Anesthesiology. 2010;113(4):892-905. doi:10.1097/ ALN.0b013e3181d960a9

27. Newman S, Wilkinson DJ, Royse CF. Assessment of early cognitive recovery after surgery using the post-operative quality of recovery scale. Acta Anaesthesiol Scand. 2014;58(2):185-191. doi:10.1111/ aas. 12234

28. Royse CF, Williams Z, Purser S, Newman S. Recovery after nasal surgery vs. tonsillectomy: discriminant validation of the postoperative quality of recovery scale. Acta Anaesthesiol Scand. 2014;58 (3):345-351. doi:10.1111/aas.12264

29. Amorim P, Lagarto F, Gomes B, et al. Neostigmine vs. sugammadex: observational cohort study comparing the quality of recovery using the Postoperative Quality Recovery Scale. Acta Anaesthesiol Scand. 2014;58(9):1101-1110. doi:10.1111/aas.12389

30. Apfel CC, Laara E, Koivuranta M, Greim CA, Roewer N. A simplified risk score for predicting postoperative nausea and vomiting: conclusions from cross-validations between two centers. Anesthesiology. 1999;91(3):693-700. doi:10.1097/00000542199909000-00022

31. Peng K, Zhang J, Chen WR, Liu HY, Ji FH. Ultrasound-guided stellate ganglion block improves gastrointestinal function after thoracolumbar spinal surgery. Clin Ther. 2017;39(11):2322-2330. doi:10.1016/j.clinthera.2017.09.008
32. Moher D, Hopewell S, Schulz KF, et al. CONSORT 2010 explanation and elaboration: updated guidelines for reporting parallel group randomised trials. BMJ. 2010;340:c869. doi:10.1136/bmj.c869

33. Hristovska AM, Duch P, Allingstrup M, Afshari A. Efficacy and safety of sugammadex versus neostigmine in reversing neuromuscular blockade in adults. Cochrane Database Syst Rev. 2017;8: CD012763. doi:10.1002/14651858.CD012763

34. Özdemir-van Brunschot DMD, Braat AE, van der Jagt MFP, et al. Deep neuromuscular blockade improves surgical conditions during low-pressure pneumoperitoneum laparoscopic donor nephrectomy. Surg Endosc. 2018;32(1):245-251. doi:10.1007/s00464-017-5670-2

35. Choi BM, Ki SH, Lee YH, et al. Effects of depth of neuromuscular block on postoperative pain during laparoscopic gastrectomy: a randomised controlled trial. Eur J Anaesthesiol. 2019;36 (11):863-870. doi:10.1097/EJA.0000000000001082

36. Boggett S, Chahal R, Griffiths J, et al. A randomised controlled trial comparing deep neuromuscular blockade reversed with sugammadex with moderate neuromuscular block reversed with neostigmine. Anaesthesia. 2020;75(9):1153-1163. doi:10.1111/anae.15094

37. Baete S, Vercruysse G, Vander Laenen M, et al. The effect of deep versus moderate neuromuscular block on surgical conditions and postoperative respiratory function in bariatric laparoscopic surgery: a randomized, double blind clinical trial. Anesth Analg. 2017;124 (5):1469-1475. doi:10.1213/ANE.0000000000001801

38. Madsen MV, Istre O, Staehr-Rye AK, et al. Postoperative shoulder pain after laparoscopic hysterectomy with deep neuromuscular blockade and low-pressure pneumoperitoneum: a randomised controlled trial. Eur J Anaesthesiol. 2016;33(5):341-347. doi:10.1097/ EJA.0000000000000360

39. Gin E, Lowen D, Tacey M, Hodgson R. Reduced laparoscopic intra-abdominal pressure during laparoscopic cholecystectomy and its effect on post-operative pain: a double-blinded randomised control trial. J Gastrointest Surg. 2021. doi:10.1007/s11605-021-04919-0

40. Royse CF, Williams Z, Ye G, et al. Knee surgery recovery: post-operative quality of recovery scale comparison of age and complexity of surgery. Acta Anaesthesiol Scand. 2014;58(6):660-667. doi:10.1111/aas. 12273
Journal of Pain Research

\section{Publish your work in this journal}

The Journal of Pain Research is an international, peer reviewed, open access, online journal that welcomes laboratory and clinical findings in the fields of pain research and the prevention and management of pain. Original research, reviews, symposium reports, hypothesis formation and commentaries are all considered for publication. The manuscript management system is completely online and includes a very quick and fair peer-review system, which is all easy to use. Visit http:// www.dovepress.com/testimonials.php to read real quotes from published authors. 